
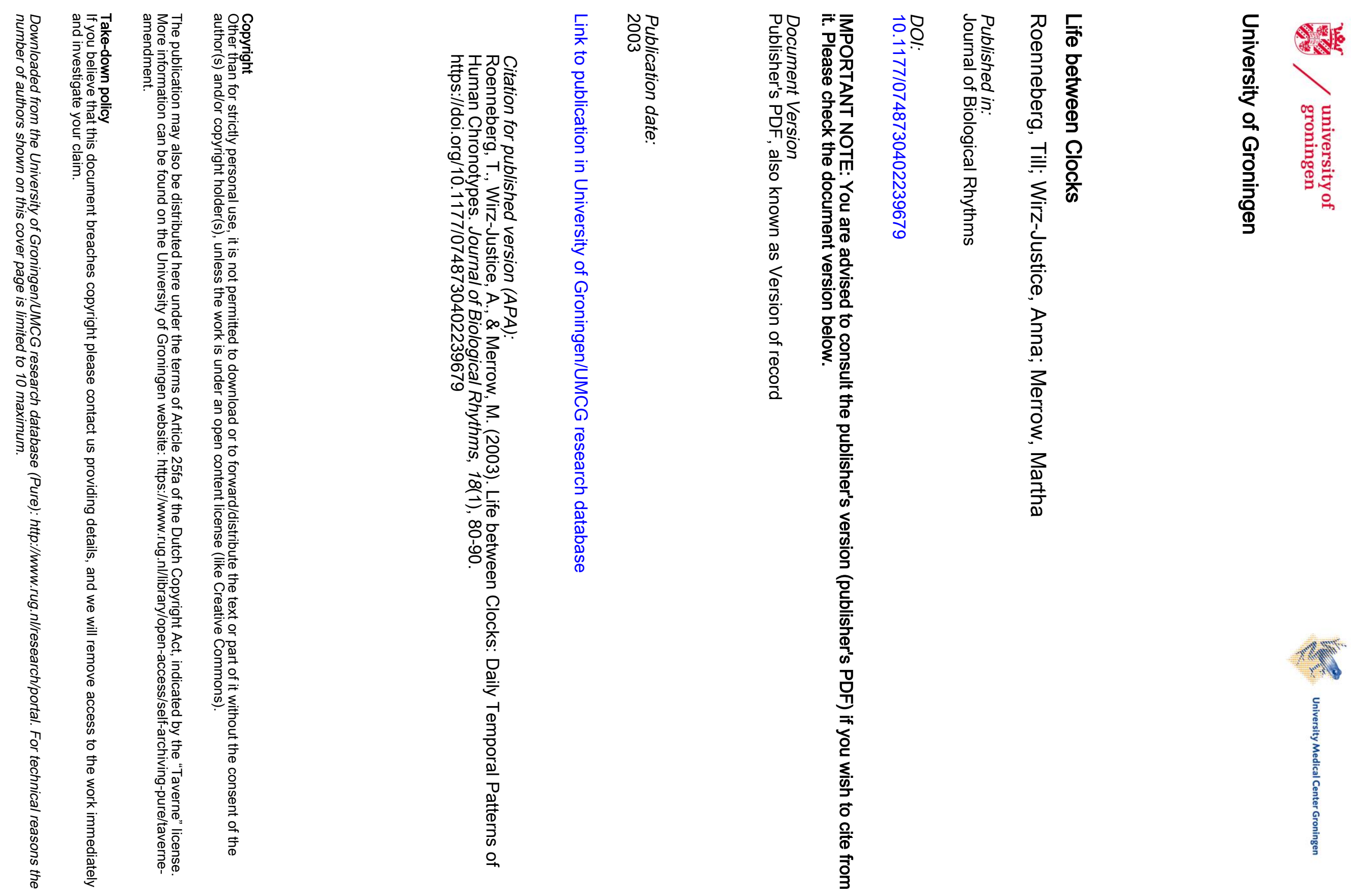


\title{
Life between Clocks: Daily Temporal Patterns of Human Chronotypes
}

\author{
Till Roenneberg, ${ }^{*, 1}$ Anna Wirz-Justice, + and Martha Merrow ${ }^{*}$ \\ *Centre for Chronobiology, Institute for Medical Psychology, Munich, Germany, \\ tCentre for Chronobiology, Psychiatric University Clinic, Basel, Switzerland
}

\begin{abstract}
Human behavior shows large interindividual variation in temporal organization. Extreme "larks" wake up when extreme "owls" fall asleep. These chronotypes are attributed to differences in the circadian clock, and in animals, the genetic basis of similar phenotypic differences is well established. To better understand the genetic basis of temporal organization in humans, the authors developed a questionnaire to document individual sleep times, self-reported light exposure, and self-assessed chronotype, considering work and free days separately. This report summarizes the results of 500 questionnaires completed in a pilot study. Individual sleep times show large differences between work and free days, except for extreme early types. During the workweek, late chronotypes accumulate considerable sleep debt, for which they compensate on free days by lengthening their sleep by several hours. For all chronotypes, the amount of time spent outdoors in broad daylight significantly affects the timing of sleep: Increased self-reported light exposure advances sleep. The timing of selfselected sleep is multifactorial, including genetic disposition, sleep debt accumulated on workdays, and light exposure. Thus, accurate assessment of genetic chronotypes has to incorporate all of these parameters. The dependence of human chronotype on light, that is, on the amplitude of the light:dark signal, follows the known characteristics of circadian systems in all other experimental organisms. Our results predict that the timing of sleep has changed during industrialization and that a majority of humans are sleep deprived during the workweek. The implications are far ranging concerning learning, memory, vigilance, performance, and quality of life.
\end{abstract}

Key words circadian, clock, human, light, sleep, chronotype

Our daily life is organized by three different clocks: a solar clock, providing light and warmer temperatures during the day; a social clock, which we see or hear first thing on a working day; and a biological clock, which we sense most vividly when jet lagged, during shift work, or when adjusting to daylight sav- ings time. When shielded from the solar and the social clock (constant conditions), the biological clock "runs free." In real life, however, circadian clocks are usually synchronized (entrained) to the $24 \mathrm{~h}$ of the solar clock (Rajaratnam and Arendt, 2001; Roenneberg and Foster, 1997). The major environmental signal for entrain-

\footnotetext{
1. To whom all correspondence should be addressed: Centre for Chronobiology, Institute for Medical Psychology, Munich, Germany; e-mail: till.roenneberg@imp.med.uni-muenchen.de.
}

JOURNAL OF BIOLOGICAL RHYTHMS, Vol. 18 No. 1, February $2003 \quad 80-90$

DOI: $10.1177 / 0748730402239679$

(C) 2003 Sage Publications

80 
ment (zeitgeber) is light that, in mammals, can reach the biological clock only through the eyes (for references, see Roenneberg and Merrow, 2002).

The circadian clock controls physiology at many levels, from gene expression to complex behaviors (e.g., sleep and performance). As with other genetic traits, circadian properties depend on specific alleles of genes (clock genes; for review, see Young and Kay, 2001). In a given population, free-running periods are distributed around a species-specific mean both in animals (Pittendrigh and Daan, 1976) and in humans (e.g., Dijk and Lockley, 2002; Klerman, 2001; Wever, 1979). These circadian differences can also be seen when the clock is entrained because the phase angle between dawn and activity onset depends on the individual free-running period (Aschoff, 1979; Duffy et al., 2001; Roenneberg and Merrow, 2000). Human preferences in the timing of sleep and wake (called "chronotypes," such as "larks" and "owls") are, at least partly, based on genetics (Ebisawa et al., 2001; Jones et al., 1999; Katzenberg et al., 1998; Toh et al., 2001).

Chronotypes have been assessed mainly by questionnaires designed to associate individuals to tendencies that were coined "morningness" or "eveningness" (Horne and Östberg, 1976). The questions used are mostly subjective, relating sleep and activity times to a personal "feeling best rhythm" (Horne and Östberg, 1976) or to the habits of others (e.g., "I get up later than most people") (Smith et al., 2002), or they ask subjects to assess hypothetical situations (e.g., "Approximately what time would you get up if you were entirely free to plan your day?") (Terman and White, 2001). These questionnaires yield plausible results, yet oddly enough, in terms of "real" behavior, they do not explicitly assess free days and workdays separately nor do they ask for actual sleep times (Putilov, 2000) or exposure to outdoor light. These details have, so far, been addressed only in a questionnaire assessing seasonality (Wirz-Justice et al., 1996, 2003).

A growing number of studies investigate the genetic basis of the human circadian clock and its relationship to abnormal sleep times using the existing questionnaires (e.g., Ebisawa et al., 2001; Jones et al., 1999; Katzenberg et al., 1998). However, determination of circadian genes in experimental organisms stems from the excellent ability to quantify circadian outputs (e.g., period length). Thus, successful genetic research into the human clock also requires a quantitative description of circadian traits. Although "morningness" scores correlate with the experimen- tally measured timing of the individual's circadian rhythms, for example, temperature, melatonin, or cortisol (Bailey and Heitkemper, 2001; Duffy et al., 1999, 2001), the genetics behind "morningness" and "eveningness" are far from straightforward (Duffy and Czeisler, 2002; Duffy et al., 1999; Vink et al., 2001).

We have developed a new questionnaire to quantitatively assess the timing of sleep within the 24-h day (sleep phase) using simple questions (see the Methods section). The results of this pilot study show that although indicated sleep times correlate well with chronotype self-assessment, they are very different between workdays and free days. Most important, phase and duration of sleep on free days strongly depend on (1) the amount of sleep obtained during the workweek and (2) the amount of time subjects spend outdoors in daylight. Thus, timing of sleep is a good indicator for the underlying chronotype when corrected for these factors.

\section{METHODS}

This article describes the results of an ongoing study, based on the first 500 completed questionnaires (see Fig. 1), which were distributed in Germany and Switzerland in high schools, universities, and the general population mainly between March and June (shift workers were excluded; for age and gender distribution, see Table 1). All questionnaires were filled in voluntarily, and subjects were informed about confidentiality and data handling on a cover page (not shown). We continue to distribute and evaluate the questionnaire in a project that searches for extreme chronotypes and their families, and the results presented here prove to be extremely stable. This first report concentrates on the temporal distribution of daily behavior and therefore does not address all questions. The temporal structure of daily life was assessed separately for workdays and free days. Subjects are explicitly asked to describe their behavior for normal circumstances, that is, without partying. In addition, subjects self-rated their chronotype category based on a description ( $0=$ extreme early, $1=$ moderate early, $2=$ slight early, $3=$ normal, $4=$ slight late, $5=$ moder ate late, 6 = extreme late; see questionnaire in Fig. 1).

A preliminary validation was carried out in a random sample of subjects $(N=30)$ who kept a sleep log for 5 weeks after completing the questionnaire. Actual and indicated sleep times on both workdays and free days correlated highly $(p<0.0001)$ with slopes around 


\section{Munich ChronoType Questionnaire (MCTQ)}

Please enter your age, gender, etc.. This information is important for our evaluations

Age: ${ }_{--------}$female male Height ${ }_{------}$Weight $_{-------}$

On work days ...

$$
\begin{aligned}
& \text { I have to get up at... } \\
& \text { I need... - } \\
& \text { I regularly wake up... before the alarm with the alarm } \\
& \text { From... _ _ _ 'clock I am fully awake }
\end{aligned}
$$$$
\text { At around... } \quad \text { o'clock, I have an energy dip }
$$

On nights before workdays, I go to bed at

$$
\text { and it then takes me... }
$$

If I get the chance, I like to take a siesta/nap ...

$$
\begin{aligned}
\text { correct } & \text { I then sleep for...--_-_---- } \min \\
\text { not correct } & \text { I would feel terrible afterwards }
\end{aligned}
$$

On free days (please only judge normal free days, i.e., without parties etc.) .. My dream would be to sleep until...

$$
\text { I normally wake up at... _-_-_o'clock }
$$

If I wake up at around the normal (workday) alarm time, I try to get back to sleep...

$$
\begin{aligned}
& \text { correct not correct } \\
& \text { if I get back to sleep, I sleep for another... _-____-_min } \\
& \text { I need ...___ min to wake up } \\
& \text { From... _-______'clock I am fully awake } \\
& \text { At around... - - } \\
& \text { On nights before free days, I go to bed at... } \\
& \text { and it then takes me... }
\end{aligned}
$$

\begin{tabular}{|c|c|c|}
\hline Description of categories: & $\begin{array}{l}\text { extreme } \\
\text { moderate } \\
\text { slight } \\
\text { slight } \\
\text { moderate } \\
\text { extreme }\end{array}$ & $\begin{array}{r}\text { early type }= \\
\text { early type }= \\
\text { early type }= \\
\text { normal type }= \\
\text { late type }= \\
\text { late type }= \\
\text { late type }=\end{array}$ \\
\hline
\end{tabular}

\begin{tabular}{|c|c|c|c|c|c|c|c|}
\hline I am... & 0 & 1 & 2 & 3 & 4 & 5 & 6 \\
\hline as a child, I was ... & 0 & 1 & 2 & 3 & 4 & 5 & 6 \\
\hline as a teenager, I was ... & .0 & 1 & 2 & 3 & 4 & 5 & 6 \\
\hline \multicolumn{8}{|c|}{ In case you are older than 65: in the middle of my life, I was } \\
\hline \multicolumn{8}{|c|}{ My parents are/were... } \\
\hline Mother ... & 0 & 1 & 2 & 3 & 4 & 5 & 6 \\
\hline Father $\ldots$ & 0 & 1 & 2 & 3 & 4 & 5 & 6 \\
\hline
\end{tabular}

If I get the chance, l like to take a siesta/nap ...

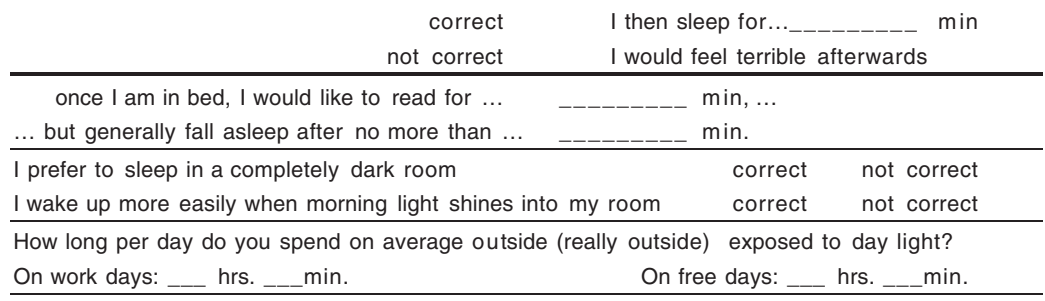

\section{Self assessmen}

After you have answered the preceding questions, you should have a feeling to which chronotype (time-of-day-type) you belong to. If for example, you like (and manage) to sleep quite a bit longer on free days than on workdays, or if you cannot get out of bed on Monday mornings, even without a Sunday-night-party, then you are more a late type. If, however, you regularly wake up and feel perky once you jump out of bed, and if you would rather go to bed early than to an evening concert then you are an early type. In the following questions, you should categorise yourself and your family members.

My siblings are/were ... (please underline Brother or $\underline{\text { Sister) }}$

Brother/Sister Brother/Sister Brother/Sister Brother/Sister Brother/Sister Brother/Siste Brother/Sister My partner (girl/boy frienc

\begin{tabular}{ccccccc}
0 & 1 & 2 & 3 & 4 & 5 & 6 \\
0 & 1 & 2 & 3 & 4 & 5 & 6 \\
0 & 1 & 2 & 3 & 4 & 5 & 6 \\
0 & 1 & 2 & 3 & 4 & 5 & 6 \\
0 & 1 & 2 & 3 & 4 & 5 & 6 \\
0 & 1 & 2 & 3 & 4 & 5 & 6 \\
0 & 1 & 2 & 3 & 4 & 5 & 6 \\
\hline \\
$\begin{array}{l}\text { friend, spouse, significant } \\
0\end{array}$ & 2 & 3 & 4 & 5 & 6
\end{tabular}

๑ 2002, Till Roenneberg and Martha Merrow, LMU Müchen.

Figure 1. The complete questionnaire. The original was distributed over two pages including a cover letter informing the volunteers about confidentiality and data handling. 
Table 1. Average timing and duration of sleep for the entire sample population and for different age groups and gender.

\begin{tabular}{lccc}
\hline & n & $\begin{array}{c}\text { Work Days } \\
\varnothing \pm \mathrm{SD}\end{array}$ & $\begin{array}{c}\text { Free Days } \\
\varnothing \pm \mathrm{SD}\end{array}$ \\
\hline Midsleep (time, h:min) & 500 & $3: 10 \pm 50^{\prime}$ & $5: 02 \pm 92^{\prime}$ \\
$\quad$ <21 years of age & 142 & $3: 00 \pm 36^{\prime}$ & $5: 54 \pm 96^{\prime}$ \\
21 to 30 years of age & 195 & $3: 18 \pm 36^{\prime}$ & $5: 12 \pm 66^{\prime}$ \\
> 30 years of age & 163 & $3: 06 \pm 66^{\prime}$ & $4: 06 \pm 84^{\prime}$ \\
Women & 278 & $3: 06 \pm 47^{\prime}$ & $4: 53 \pm 86^{\prime}$ \\
Men & 222 & $3: 15 \pm 53^{\prime}$ & $5: 12 \pm 97^{\prime}$ \\
Sleep duration (h:min) & 500 & $7: 22 \pm 69^{\prime}$ & $8: 27 \pm 92^{\prime}$ \\
$<21$ years of age & 142 & $7: 24 \pm 66^{\prime}$ & $9: 18 \pm 90^{\prime}$ \\
21 to 30 years of age & 195 & $7: 12 \pm 54^{\prime}$ & $8: 12 \pm 96^{\prime}$ \\
$>$ 30 years of age & 163 & $7: 30 \pm 80^{\prime}$ & $8: 00 \pm 72^{\prime}$ \\
Women & 278 & $7: 29 \pm 71^{\prime}$ & $8: 32 \pm 87^{\prime}$ \\
Men & 222 & $7: 12 \pm 64^{\prime}$ & $8: 20 \pm 98^{\prime}$ \\
\hline
\end{tabular}

NOTE: Among the youngest age group, only 7 subjects were between 6 and 15 years; among the adult category, only 15 were older than 65 years.

1 and an interception near the origin. Because of the excellent agreement between indicated sleep times and those assessed by sleep logs, they are regarded as a quantitative assessment of sleep phase while the subjective self-rating of chronotype represents a qualitative assessment.

The term "chronotype" refers to sleep phase and not to sleep duration, but individuals vary in both. For an investigation of chronotypes, it is necessary to define a single phase reference point (e.g., onset, midpoint, or offset). Sleep onset was calculated by subtracting the indicated time it took to fall asleep from the given bedtime. We chose the midpoint between sleep onset and wake up (midsleep) as the phase reference point for sleep (see also Benoit et al., 1981). Midsleep has also been reported as the best phase anchor point for melatonin onset (Terman et al., 2001).

\section{RESULTS AND DISCUSSION}

\section{Quantitative Assessment of Chronotype by Sleep Phase}

Midsleep times show a normal distribution for different age groups both on workdays and free days. During the workweek (Fig. 2A), the phase of midsleep is latest for young adults (21 to 30 years of age), with adults $(>30)$ and adolescents $(<21)$ sleeping slightly earlier (see Table 1). The distributions are drastically different on free days (Fig. 2B): All three become wider and shift to later hours. Adolescents delay their sleep on average by almost $3 \mathrm{~h}$, young adults by approxi- mately $2 \mathrm{~h}$, and adults by $1 \mathrm{~h}$. Analysis of variance (two-way ANOVA) shows a significant difference in midsleep between workdays and free days and between the different age groups $(p<0.0001$; interaction: $p<0.0001)$. ANOVA subanalyses reveals the difference between the age groups to be more significant on free days $(p<0.0001)$ than on workdays $(p<0.001)$. The fact that self-selected sleep of adolescents occurs much later than in other ages has already been shown (Carskadon et al., 2001, 1999), and the consequences on performance and health have been well documented (Fallone et al., 2001).

Sleep duration also differs markedly between workdays and free days (Fig. 2C). On average, subjects sleep $1 \mathrm{~h}$ longer on free days, with extreme individuals sleeping as long as $12 \mathrm{~h}$. Women sleep only slightly longer than do men (see Table 1; ANOVA shows this difference to be significant only on workdays, $p=0.002$ ), and men sleep on average slightly later than do women (see Table 1), although ANOVA shows this difference to be not statistically significant.

\section{Qualitative Assessment of Chronotype}

Self-rating of chronotype (for categories, see the Methods section) is also normally distributed in our sample population (Fig. 2D, gray bars; overall average of $3.4 \pm 1.4)$. "Normal" chronotypes are the largest group (27\%), and "extreme late" is chosen threefold more often than "extreme early." Only 10 subjects qualify themselves as chronotype 0 , and quantitatively, 4 fall into the earliest midsleep category (black bars in Fig. 2D). Overall, self-awareness of chronotype is in excellent agreement with the quantitatively assessed sleep times (onset, offset, and midsleep correlate significantly with qualitative chronotype; $p<$ 0.001). Predictably, wake up on workdays, being dictated by the social clock, has the weakest correlation, and midsleep on free days has the highest ( $r=0.3 \mathrm{vs.}$ $r=0.6$; see also Bailey and Heitkemper, 1991). The gender differences in the quantitative assessment (see Table 1) are also present in the qualitative assessment. Women qualify themselves as slightly earlier chronotypes than do men $(3.2 \pm 1.4$ vs. $3.5 \pm 1.3$; ANOVA: $p=0.05)$.

\section{The Average Days of Different Chronotypes}

Qualitative and quantitative chronotyping lead to very similar results on both workdays and free days (Fig. 3). Error bars in this and other figures represent 

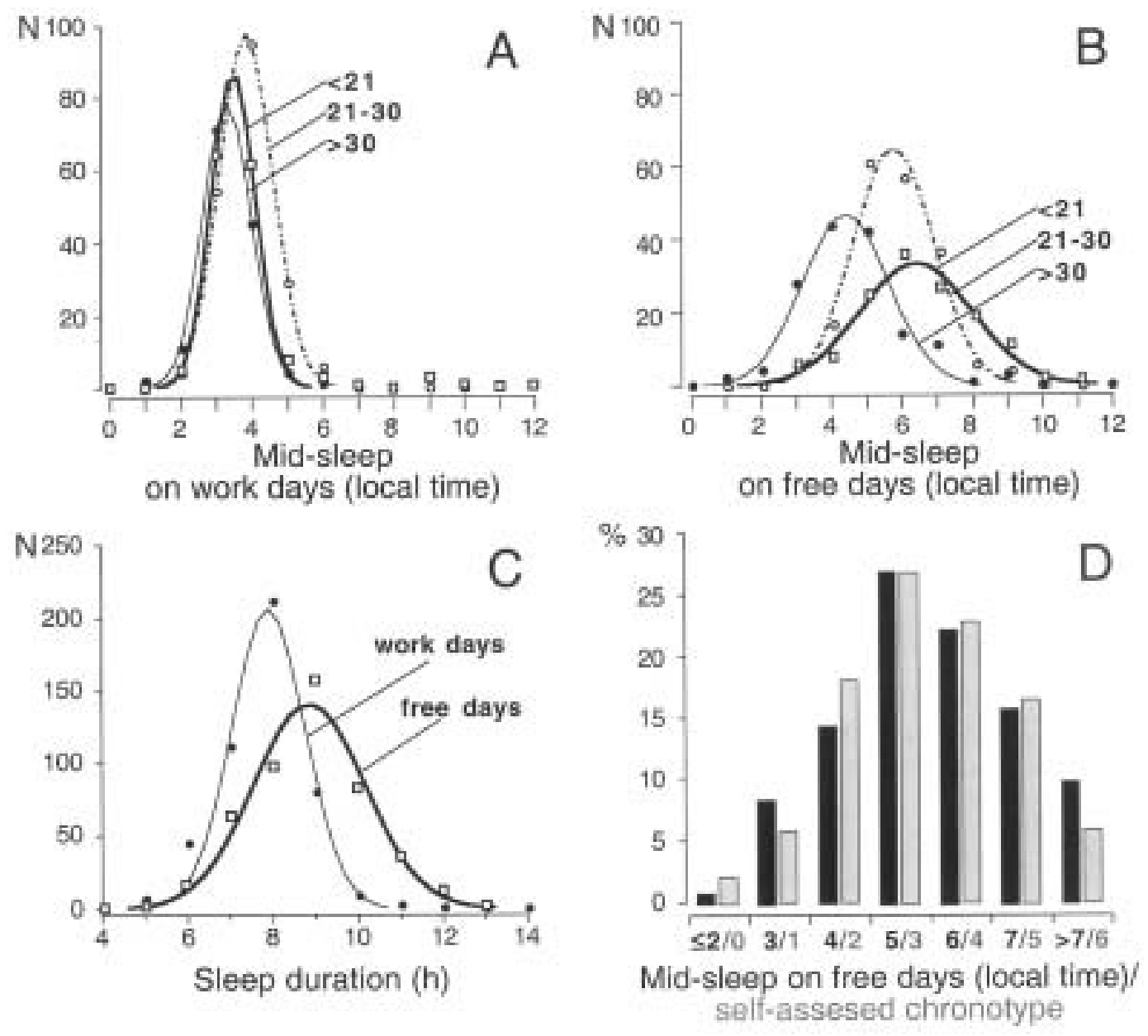

Figure 2. Distributions of midsleep phase, sleep duration, and chronotype. Panels A and B show the distribution of midsleep on workdays and free days, respectively, for different age groups (adolescents: $<21$, young adults: 21-30, and adults: $>30$ ). All distributions can be fitted with a Gauss curve with high significance (see text). Like the distribution of timing of sleep, the distribution of sleep duration also changes drastically between workdays and free days ( $C$; all ages). Panel $D$ compares the distribution of midsleep on free days (black bars) and of self-assessed chronotypes (gray bars, see Methods section). The lowest and highest bins of midsleep on free days represent $\leq 2 \mathrm{AM}$ and > 7 AM, respectively; intermediary bins exclude the lower and include the upper limit.

standard deviations and not SEM. Small error bars and good correlations are to be expected in Fig. 3D because sleep times on free days, by definition, determine the phase of midsleep (quantitative chronotypes). The tight statistics in the other panels, however, show the homogeneity of sleep behavior within the respective chronotypes. During the workweek (Fig. 3A, B), early chronotypes fall asleep almost $2 \mathrm{~h}$ before the late chronotypes do. This difference shrinks down to $30 \mathrm{~min}$ at wake up, leading to a systematic shortening of sleep from early to late chronotype. Chronotype-specific differences are much more pronounced on free days (Fig. 3C, D).

The graphs in Fig. 3 also show how long individuals take to feel fully awake. This time is comparable to the so-called sleep inertia, characterized by decreased vigilance and performance (Ferrara and De Gennaro, 2000). It spans the transition from sleep to the fully awake state and is different from the time to wake up. While the latter is relatively short, the former can last hours. On workdays, the time to feel fully awake increases with later chronotypes but runs parallel to sleep onset; that is, the shorter the sleep, the longer the sleep inertia. On free days, sleep inertia is independent of chronotype or sleep duration (parallel to wakeup in Fig. 3C, D) and lasts on average $1 \mathrm{~h}$.

The large differences in chronotype-specific sleep behavior between workdays and free days become even more apparent when subjects' average days are represented as 24-h dials (Fig. 4). While average workdays (top row) are similarly structured for all chronotypes (in spite of chronotype-dependent sleep 


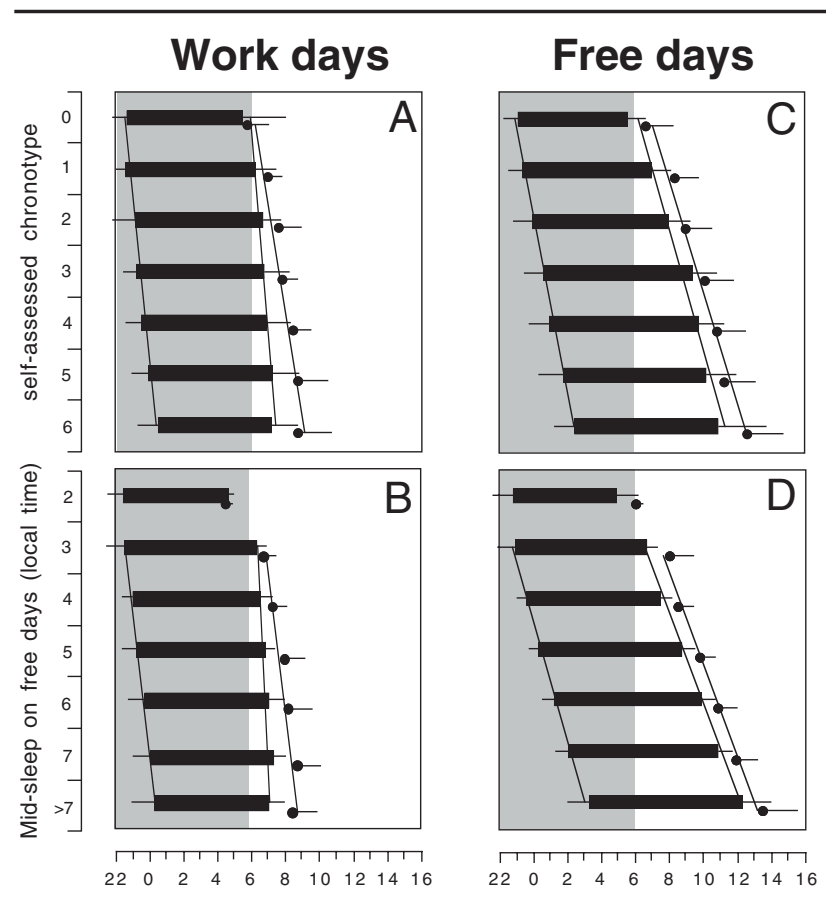

Figure 3. Sleep patterns of different chronotypes grouped by qualitative (A, C) and quantitative (B, D) assessment (see Methods section). Workdays are shown on the left, and free days are shown on the right. Sleep periods (onset to wake up) are drawn as horizontal bars. The bins for grouping quantitative chronotypes by phase of midsleep are the same as in Fig. 1D. Thin horizontal lines represent standard deviation. Dots represent the times when subjects feel fully awake (end of sleep inertia). Regression lines are drawn through sleep onset, wake up, and the times when subjects feel fully awake. Due to their small number $(n=4)$ and unusually short sleep duration, extreme early chronotypes assessed quantitatively by indicated times were excluded from the regressions in panels B and D. All regressions in the respective panels are highly significant (regression coefficients, $r$, for the binned/raw data are given for sleep onset, wake up, and the times when subjects are "fully awake"): (A): 0.97/0.44, 0.92/0.30, 0.95/0.42; (B): 0.99/0.55, 0.88/0.60, 0.93/0.55; (C): 0.99/0.53, 0.97/0.32, 0.99/0.49; (D): 0.98/0.87, 0.997/0.93, 0.99/0.81.

onset and sleep inertia), it drastically changes on free days (bottom row). The immediate time to wake up (black areas) increases from early to late chronotype both on workdays and free days. The only factor independent of chronotype is average time of day when subjects experience a midday energy dip: This, however, differs greatly between workdays (13.4 $\pm 2.3 \mathrm{~h})$ and free days $(15.4 \pm 2.4 \mathrm{~h})$.

\section{Chronotypes and Sleep Duration}

A chronotype-specific sleep-onset time exists even on workdays despite similar wake-up times for all chronotypes (Figs. 3 and 4), indicating that the social clock is not a strong enough zeitgeber to advance the circadian clock or the phase of sleep. Later chronotypes, therefore, accumulate a considerable sleep debt during the workweek, which they compensate for on free days (see diverging regression lines in Fig. 5). Except for the 4 extreme early subjects, all chronotypes maintain a weekly average sleep duration of $7 \mathrm{~h}$, $41 \mathrm{~min} \pm 59 \mathrm{~min}$ (chronotype-specific weekly averages are indicated as bars).

In contrast to the loss of sleep of late chronotypes during the workweek, early chronotypes tend to suffer from sleep loss on free days when they comply with social pressures (from the majority, who are late chronotypes) to stay up late. Subjects were asked to state both the time at which they actually wake up and the time until when they would like to sleep on free days. On average, extreme early chronotypes (judged by midsleep) would like to sleep $3.5 \mathrm{~h}$ more than they actually do. This difference steadily shrinks to an average of $6 \mathrm{~min}$ in extreme late chronotypes. Whereas the social clock wakes late chronotypes too early on workdays, the biological clock wakes early chronotypes too early on free days.

The most recent sleep study in the United States $(N=$ 1000 , older than 18 years) investigated a large number of sleep variables, although timing of sleep was not investigated (see www.sleepfoundation.org). The ratios of sleep duration between workdays and free days and between men and women are comparable with our study, but U.S. citizens, on average, sleep less than the European population investigated here (workweek: $6 \mathrm{~h}, 54 \mathrm{~min}$, compared to $7 \mathrm{~h}, 22 \mathrm{~min}$; weekends: $7 \mathrm{~h}, 30 \mathrm{~min}$, compared to $8 \mathrm{~h}, 27 \mathrm{~min}$; see Table 1). Thus, cultural norms additionally modify sleep-wake behavior.

\section{Zeitgeber Strength and Phase of Entrainment}

The phase of entrainment not only depends on the free-running period (see the opening section of this article) but also on the strength of the zeitgeber (i.e., on the difference between daytime and nighttime light intensity). Mid-daytime light intensities outdoors range from 10,000 to many tens of thousands lux depending on cloud cover, while most indoor environments are generally below 400 lux. Subjects were, therefore, asked to indicate their average duration of exposure to outdoor light on workdays and free days. A previous study comparing self-reported light exposure and daily light logs has shown good consistency (Wirz-Justice et al., 1996). 


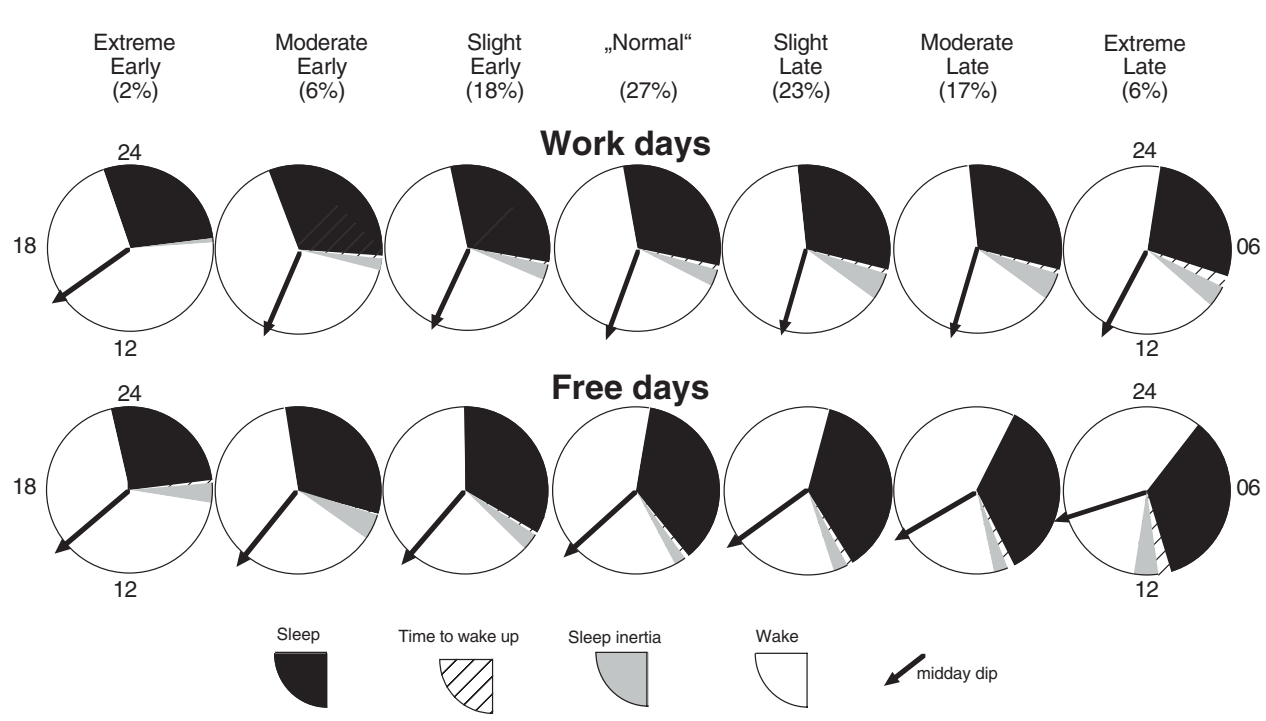

Figure 4. The average day of different chronotypes on workdays (top row) and free days (bottom row) represented as 24-h dials. Different patterns represent sleep, time to wake up, sleep inertia (the time it takes to become fully awake), and wake time; arrows show the average times of the "midday dip."

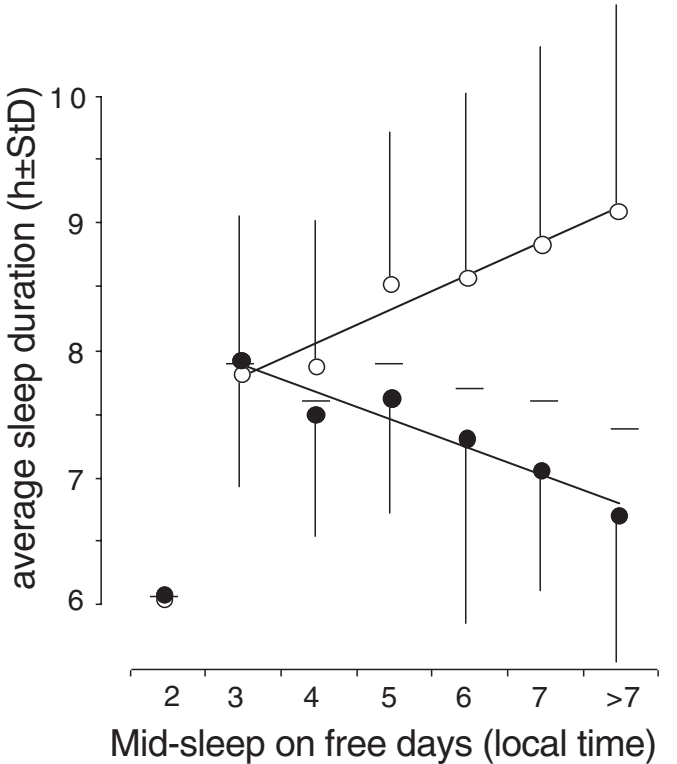

Figure 5. Sleep duration on free days and workdays. Bars represent standard deviation. The 4 extreme early chronotypes were not included in the regressions. Results for regression through binned/raw data: workdays: $r=-0.97 /-0.26(p<0.001)$; free days: $r=$ $0.98 / 0.29(p<0.001)$. The chronotype-specific sleep durations averaged over the week are indicated as horizontal bars.

On average, subjects spend $1.5 \mathrm{~h}$ outdoors on workdays and almost $4 \mathrm{~h}$ on free days, with extremes of $5 \mathrm{~min}$ and $8 \mathrm{~h}$, respectively. The average phase of midsleep on free days correlates significantly with the average weekly self-reported light exposure $(5 \times$

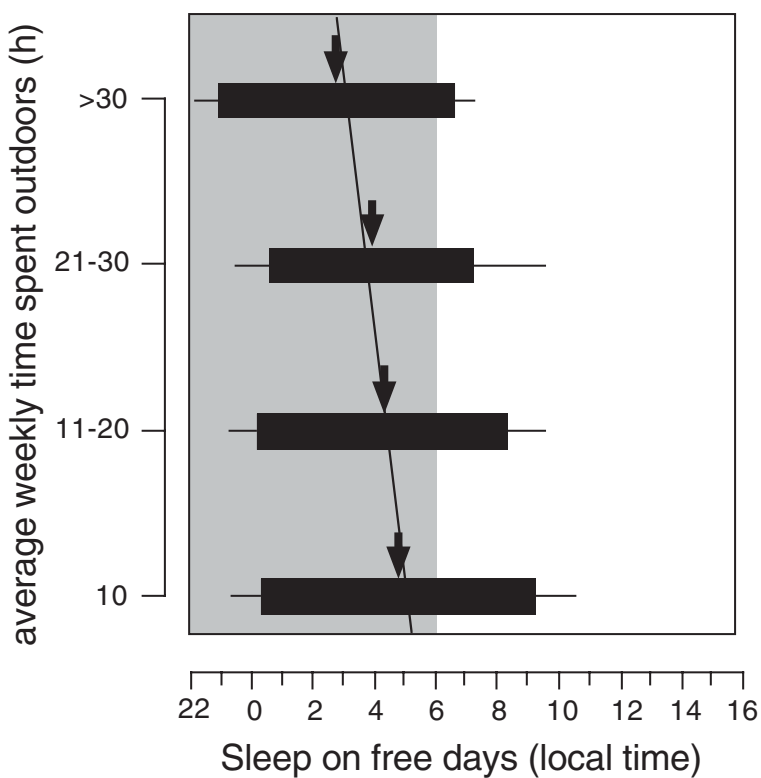

Figure 6. Weekly time spent outside in daylight and sleep phase on free days. Horizontal bars represent sleep (onset to wake up with standard deviations; arrows indicate midsleep) binned into groups based on the indicated times in actual hours (ranging from less than $10 \mathrm{~h}$ per week to more than $30 \mathrm{~h}$ per week). Linear regression line represents results based on midsleep of the binned data $(r=-0.97$; significance of correlation through raw data: $p=0.005)$.

workday $+2 \times$ free day; Fig. 6 ); the longer the exposure to outdoor light, the more advanced the sleep period. Subjects who spend more than $30 \mathrm{~h} /$ week outdoors position their sleep almost $2 \mathrm{~h}$ earlier than those who 

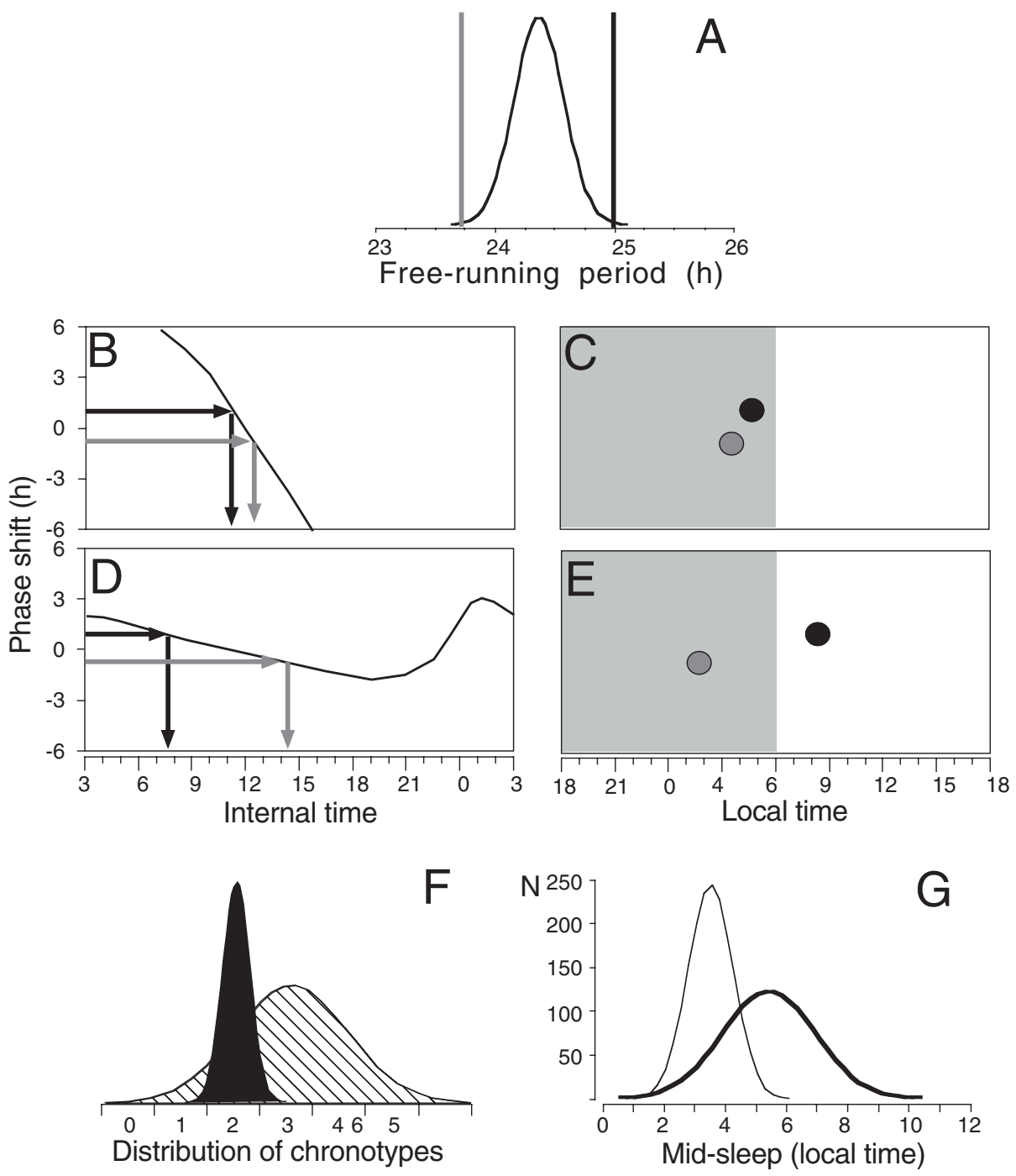

Figure 7. Theoretical considerations. The distribution of circadian periods measured in humans in isolation shows a peak at periods longer than $24 \mathrm{~h}$ (A; the gray line marks the extreme short end of the period distribution and the black line the extreme long end). The responses of circadian systems to light signals at different times of the endogenous cycle can be illustrated with phase response curves (PRCs) that are steeper the stronger the light signal (B, D). The PRCs drawn here exemplify hypothetical phase shifts caused by a light centered on its midpoint (e.g., light from 6 to $18 \mathrm{~h}$ is represented by internal time 12 and would not lead to a phase shift regardless of its intensity). The necessary phase shifts to entrain circadian clocks that deviate from $24 \mathrm{~h}$ can be achieved only if the circadian clock positions itself at a given phase angle in respect to the light signal (gray and black arrows correspond to the extreme periods indicated in A). These phases of entrainment (dots exemplify the daily temperature minima; gray and black dots correspond to the extreme periods indicated in A) are shown in panels $\mathrm{C}$ and $\mathrm{E}$ for a strong and weak zeitgeber, respectively, in relationship to an average day with sunlight from $6 \mathrm{AM}$ to $6 \mathrm{PM}$. The distribution of human periods and the different phase response curves for strong and weak zeitgebers lead to different chronotype distributions (F; black for strong and hatched for weak zeitgeber). In panel G, the distributions shown in Fig. 1A and B for different age groups are redrawn for the entire sample population. For details, see text.

spend $10 \mathrm{~h} /$ week or less. Because late chronotypes have less time to spend outdoors in daylight, we also correlated midsleep to the relative time spent outdoors (percentage of remaining daylight after wake up), which gave comparable results (not shown).

Judged by the self-reports of light exposure, zeitgeber strength affects the phase of entrainment in humans, as it does in other organisms. On average, each additional hour spent outdoors per day corresponds to an advance of sleep of almost $30 \mathrm{~min}$. This phase dependency is much more likely to be caused by the light exposure than the possibility that people are more active when outdoors because activity has been shown to have little effect on the circadian clock 
in humans (Beersma and Hiddinga, 1998) while the timing of (strong outdoor) light has been shown to affect the phase of sleep (Okudaira et al., 1983; Savides et al.,1986). The averages and the trend shown in Fig. 6 are consistent with the majority of individuals whose clock has to be advanced each day (see discussion in the next section). Individuals whose clock has to be delayed by the zeitgeber would show the opposite effect; that is, they would entrain at a later phase with increasing light exposure.

\section{Chronotypes in the Context of Circadian Theory}

In this section, we hypothesize on how the described preliminary results on light and chronotype distribution can be explained by circadian formalisms relating the free-running period to the phase of entrainment. The free-running period measured in humans who are shielded from the solar and the social clock is influenced by behavior (switching lights on and off or closing eyes during sleep). Initial studies (Wever, 1979), disregarding behavioral influences, and studies of blind people (Klerman, 2001) showed the human circadian period to be close to $25 \mathrm{~h}$. More recent studies that controlled for behavior (Dijk and Lockley, 2002; Klerman, 2001) or forced desynchrony protocols (Beersma and Hiddinga, 1998; Czeisler et al., 1999) have revealed an intrinsic, free-running period to be very close to $24 \mathrm{~h}$. These protocols are, however, neither relevant for the evolution of the circadian clock nor for the everyday life described in our questionnaires. To put our results into the context of circadian theory, we therefore based our theoretical considerations on a distribution of circadian periods that includes the influences of behavior (Fig. 7A) with a mean between 24 and $25 \mathrm{~h}$.

According to well-proven circadian mechanisms, the characteristics of entrainment by light (e.g., its phase relationship to the zeitgeber) depend on the fact that light shifts the circadian phase by different amounts and in different directions depending on when the circadian clock is exposed to light. Using single light exposures in otherwise constant conditions, so-called phase response curves (PRCs) can be established that indicate for each circadian time how much the clock is advanced (by definition, a positive value) or delayed (by definition, a negative value). These experiments have also been performed with humans (see discussion and references for human PRCs in Beersma and Daan, 1993). Depending on intensity and duration of the light signal, circadian systems respond more or less strongly, leading to steeper or flatter PRCs (see hypothetical curves in Fig. 7B and D).

The phase of entrainment depends on how much and in what direction the free-running period deviates from $24 \mathrm{~h}$, that is, how much the daily light signal has to advance or delay the clock. Thus, the phase of entrainment depends both on the strength of the zeitgeber and on the individual's free-running period. The extremes of the hypothetical period distribution (Fig. 7A) are indicated by the black and the gray line for long and short periods, respectively. For entrainment to a 24-h day, subjects with a period shorter than $24 \mathrm{~h}$ have to be delayed, while those with a longer period have to be advanced by the daily light signals (see black and gray horizontal arrows in Fig. 7B, D). When the zeitgeber is strong (steep PRC in Fig. 7B), the phases of the circadian clock at which light achieves these necessary phase shifts are close for the two extremes (see black and gray arrows in Fig. 7B), resulting in a narrow chronotype distribution (black area in Fig. 7F). With a weak zeitgeber (flat PRC curve in Fig. 7D), the phases for the extremes are farther apart and the chronotype distribution is wide (hatched area in Fig. 7F). Thus, via a PRC, the period distribution can be translated into a chronotype distribution, indicating that well-established circadian principles apparently also apply to humans.

Because the zeitgeber does not move toward the circadian clock, the clock has to move toward the zeitgeber. If light, for example, has to affect the circadian clock at an early phase (internal time) to achieve entrainment, the daily program has to settle at a later local time. To demonstrate the phase relationships between the biological and the solar clock, the times of the temperature minimum (approximately $2 \mathrm{~h}$ before wake up; Dijk and Lockley, 2002) are drawn in panels $\mathrm{C}$ and $\mathrm{E}$ of Fig. 7. Subjects with a short free-running period (gray dots) will be entrained so that their temperature minimum lies earlier than those with a long period (black dots). With decreasing zeitgeber strength, the difference increases (Fig. 7C vs. Fig. 7E).

The phase distribution on free days for the entire sample population (thick line in Fig. 7G, combining the distributions shown in Fig. 2A, B) is similar to the broad distribution for a weak zeitgeber (Fig. 7F). Because exposure to outdoor light affects the phase of entrainment (Fig. 6), a rural lifestyle would have favored a narrower chronotype distribution (similar to the black distribution in Fig. 7F). We are currently conducting a survey, including the use of actimeters 
with integrated light meters, addressing this prediction. Although this narrower distribution is similar to the distribution established from the questionnaires for workdays (thin curve in Fig. 7G), there is a significant difference between the two. While one reflects entrainment of biological clocks by a strong zeitgeber, the other reflects the effect of the social clock.

\section{CONCLUSIONS}

Proverbs praising early chronotypes are abundant, but the results shown here indicate that worm catchers are rare birds in modern society. Paradoxically, most work schedules are still tailored for this lark minority (note the small differences between workdays and free days for early chronotypes in Figs. 3 and 4). Experiments in microorganisms, plants, and animals (Roenneberg and Merrow, 2002), as well as humans (Beersma and Daan, 1993; Czeisler, 1995), show that light is the major zeitgeber for the circadian clock, and our results indicate that the broader and later distribution of chronotypes is, at least partly, due to the absence of a strong zeitgeber in modern society. There are major consequences of a redistribution of circadian phase. Large portions of society suffer from too little sleep during the workweek (Figs. 3, 4, and 5). Although the subjects contributing to this study average $7 \mathrm{~h}, 41 \mathrm{~min}$, of sleep per day, many do so only by spending almost half of their free days asleep (Fig. 2B). Sleep debt and long sleep inertia times are known to decrease attention, performance, mood (Dinges et al., 1997), and memory consolidation (Karni and Sagi, 1993). The consequences are multifarious, ranging from higher accident rates due to increased sleep inertia and decreased vigilance (Bonnet and Arand, 1995) to learning deficits in adolescents due to disturbed sleep patterns (Carskadon et al., 2001).

Our results are also relevant for refining the search for the genes responsible for circadian timing. Chronotype, as described in this study, reflects both genetic and behavioral components as well as (selfreported) light exposure. When the genetic contribution to different chronotypes is approached by searching for subjects and families with extreme circadian qualities, it is important to consider both individual light exposure and sleep debt accumulated on workdays. So far, this is the first questionnaire that allows both. The search for extreme chronotypes will yield much cleaner phenotypes when a phase distribution of midsleep is calculated for a theoretical day of $12-\mathrm{h}$ exposure to outdoor light and no sleep debt accumulated during the workweek. Those subjects who remain extreme chronotypes after recalculation will be good candidates for genetic evaluation.

Although this first pilot study does not presume to be an entirely representative sample, it covers a wide age range. Predictions arising from the data set can be (and are being) tested in independent populations. They show how the temporal structure of our daily life is influenced by the complex interactions between three clocks: the sun, the social clock, and the biological clock.

\section{ACKNOWLEDGMENTS}

We thank Puay-Luan Hong for analyzing the first third of the questionnaires with SPSS to find the questions relevant for the determination of chronotype. We are grateful to Serge Daan for excellent suggestions on the manuscript. Our work is supported by the Eppendorf Company, Hamburg; the DFG; the Dr.Meyer-Struckmann-Stiftung; and by grant QLG3CT2002-01829 (BrainTime) from the European Commission.

\section{REFERENCES}

Aschoff J (1979) Circadian rhythms: General features and endocrinological aspects. In Endocrine Rhythms, D. Krieger, ed, pp 1-60, Raven Press, New York.

Bailey SL and Heitkemper MM (1991) Morningnesseveningness and early-morning salivary cortisol levels. Biol Psychol 32:181-192.

Bailey SL and Heitkemper MM (2001) Circadian rhythmicity of cortisol and body temperature: Morningnesseveningness effects. Chronobiol Int 18:249-261.

Beersma DGM and Daan S (1993) Strong or weak phase resetting by light pulses in humans? J Biol Rhythms 8:340-347.

Beersma DGM and Hiddinga AE (1998) No impact of physical activity on the period of the circadian pacemaker in humans. Chronobiol Int 15:49-57.

Benoit O, Foret J, Merle B, and Bouard G (1981) Diurnal rhythm of axillary temperature in long and short sleepers: Effects of sleep deprivation and sleep displacement. Sleep 4:359-365.

Bonnet MH and Arand DL (1995) We are chronically sleep deprived. Sleep 18:908-911.

Carskadon MA, Acebo C, and Seifer R (2001) Extended nights, sleep loss, and recovery sleep in adolescents. Arch Ital Biol 139:301-312.

Carskadon MA, Labyak SE, Acebo C, and Seifer R (1999) Intrinsic circadian period of adolescent humans mea- 
sured in conditions of forced desynchrony. Neurosci Lett 260:129-132.

Czeisler CA (1995) The effect of light on the human circadian system. In Circadian Clocks and Their Adjustments, DJ Chadwick and K Ackrill, eds, pp 254-302, John Wiley, Chichester, UK.

Czeisler CA, Duffy JF, Shanahan TL, Brown EN, Mitchel JF, Rimmer DW, Ronda JM, Silva EJ, Allan JS, Emens JS, et al. (1999) Stability, precision, and near-24-hour period of the human circadian pacemaker. Science 284:2177-2181.

Dijk D-J and Lockley SW (2002) Functional genomics of sleep and circadian rhythm. Invited review: Integration of human sleep-wake regulation and circadian rhythmicity. J Appl Physiol 92:852-862.

Dinges DF, Pack F, Williams K, Gillen KA, Powell JW, Ott GE, Aptowicz C, and Powell JW (1997) Cumulative sleepiness, mood disturbance, and psychomotor vigilance performance decrements during a week of sleep restricted to 4-5 hours per night. Sleep 20:267-277.

Duffy JF and Czeisler CA (2002) Age-related change in the relationship between circadian period, circadian phase, and diurnal preference in humans. Neurosci Lett 318:117120.

Duffy JF, Dijk DJ, Hall EF, and Czeisler CA (1999) Relationship of endogenous circadian melatonin and temperature rhythms to self-reported preference for morning or evening activity in young and older people. J Investig Med 47:141-150.

Duffy JF, Rimmer DW, and Czeisler CA (2001) Association of intrinsic circadian period with morningnesseveningness, usual wake time, and circadian phase. Behav Neurosci 115:895-899.

Ebisawa T, Uchiyama M, Kajimura N, Mishima K, Kamei Y, Katoh M, Watanabe T, Sekimoto M, Shibui K, Kim K, et al. (2001) Association of structural polymorphisms in the human period3 gene with delayed sleep phase syndrome. EMBO Rep 2:342-346.

Fallone G, Acebo C, Arnedt JT, Seifer R, and Carskadon MA (2001) Effects of acute sleep restriction on behavior, sustained attention, and response inhibition in children. Percept Mot Skills 93:213-229.

Ferrara M and De Gennaro L (2000) The sleep inertia phenomenon during the sleep-wake transition: Theoretical and operational issues. Aviat Space Environ Med 71:843848.

Horne JA and Östberg O (1976) A self-assessment questionnaire to determine morningness-eveningness in human circadian rhythms. Int J Chronobiol 4:97-110.

Jones CR, Campbell SS, Zone SE, Cooper F, DeSano A, Murphy PJ, Jones B, Czajkowski L, and Ptacek LJ (1999) Familial advanced sleep-phase syndrome: A shortperiod circadian rhythm variant in humans. Nat-Med 5:1062-1065.

Karni A and Sagi D (1993) The time course of learning a visual skill. Nature 365:250-252.

Katzenberg D, Young T, Finn L, Lin L, King DP, Takahashi JS, and Mignot E (1998) A CLOCK polymorphism associated with human diurnal preference. Sleep 21:569-576.
Klerman EB (2001) Non-photic effects on the circadian system: Results from experiments in blind and sighted individuals. In Zeitgebers, Entrainment and Masking of the Circadian System, K Honma and S Honma, eds, pp 155-169, Hokkaido University Press, Sapporo, Japan.

Okudaira N, Kripke DF, and Webster JB (1983) Naturalistic studies of human light exposure. Am J Physiol 245:R613R615.

Pittendrigh CS and Daan S (1976) A functional analysis of circadian pacemakers in nocturnal rodents. I. The stability and lability of circadian frequency. J Comp Physiol A 106:223-252.

Putilov A (2000) Association of the circadian phase with two morningness-eveningness scales of an enlarged version of the sleep-wake pattern assessment questionnaire. Arbeitswiss betriebl Praxis 17:317-322.

Rajaratnam SMW and Arendt J (2001) Health in a 24-h society. The Lancet 358:999-1005.

Roenneberg T and Foster RG (1997) Twilight times-Light and the circadian system. Photochem Photobiol 66:549561.

Roenneberg T and Merrow M (2000) Circadian light input: Omnes viae Romam ducunt. Curr Biol 10:R742-R745.

Roenneberg T and Merrow M (2002) Light reception: Discovering the clock-eye in mammals. Curr Biol 12:R163R165.

Savides TJ, Messin S, Senger C, and Kripke DF (1986) Natural light exposure of young adults. Physiol Behav 38:571574.

Smith CS, Folkard S, Scmieder RA, Parra LF, Spelten E, Almiral H, Sen RN, Sahu S, Perez LM, and Tisak J (2002) Investigation of morning-evening orientation in six countries using the preference scale. Person Indiv Diff 32:949-968.

Terman JS, Terman M, Lo ES, and Cooper TB (2001) Circadian time of morning light administration and therapeutic response in winter depression. Arch Gen Psychiatry Res 58:69-75.

Terman M and White TM (2001) Morningness-Eveningness Questionnaire-Self-assessment version (AutoMEQSA). Retrieved from www.cet.org

Toh KL, Jones CR, He Y, Eide EJ, Hinz WA, Virshup DM, Ptacek LJ, and Fu YH (2001) An hPer2 phosphorylation site mutation in familial advanced sleep phase syndrome. Science 291:1040-1043.

Vink JM, Groot AS, Kerkho GA, and Boomsma DI (2001) Genetic analysis of morningness and eveningness. Chronobiol Int 18:809-822.

Wever R (1979) The Circadian System of Man, Springer, Berlin. Wirz-Justice A, Graw P, Kräuchi K, Sarrafzadeh A, English J, Arendt J, and Sand L (1996) "Natural" light treatment of seasonal affective disorder. J Aff Disord 37:109-120.

Wirz-Justice A, Graw P, Kräuchi K, and Wacker H-R (2002) Seasonality in affective disorders in Switzerland. Act Psychia Scand (Suppl). Forthcoming.

Young MW and Kay SA (2001) Time zones: A comparative genetics of circadian clocks. Nature Reviews Genetics 2:702-715. 